\title{
Comparative Study of Hydrogen Uptake and Diffusion in ODS Steels
}

Malitckii E. a, Yagodzinskyy Y.a, Ganchenkova M.a, ${ }^{\mathrm{a}}$, Binyukova S.

Hänninen, H. ${ }^{\mathrm{a}}$, Lindau R.c, Vladimirov P.c, Moeslang A.c

${ }^{a}$ School of Engineering, Aalto University, Finland,

b National Research Nuclear University, Moscow, Russian Federation,

c Karlsruhe Institute of Technology, Institute for Applied Materials, Germany

Aalto University

Acknowledgments: The research has been supported in part by the Academy of Finland, Doctoral Programme for Nuclear Engineering and Radiochemistry (YTERA, Finland), and by the Federal Target Program contract \#14.740.11.1130 from the Russian Ministry of Education and Science.

\section{Abstract}

3 In this work ODS steels and some of their matrix material counterparts, namely, ODS-EUROFER, EUROFER 97, and PM200o are studied in terms of their interaction with hydrogen at ambient temperature.

Hydrogen uptake and effective activation energy of its diffusion and trapping are calculated from the thermal desorption spectra obtained for EUROFER 97, ODS-EUROFER, and PM200o steels.

It is shown that embedding of ODS nanoparticles lead to significant increase of hydrogen uptake compared to the conventional ferritic steel, such as EUROFER 97.

\& It is shown that hydrogen in ODS-EUROFER has the effective diffusion activation energy, which is 0.08 and $0.06 \mathrm{eV}$ larger than that in EUROFER 97 and PM200o, respectively.

\& The high-temperature components of the TDS peaks in the studied ODS steels are suggested to reflect hydrogen de-trapping from the oxide nanoparticles.

\section{Experimental}

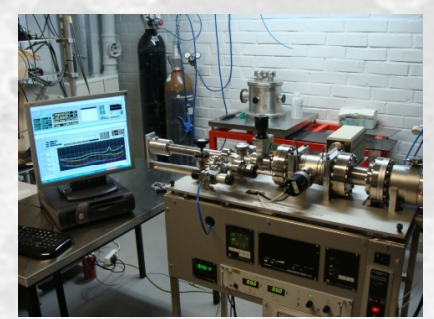

8. TDS apparatus was designed and assembled at Laboratory of Engineering Materials of Aalto University:

- linear heating from $\mathrm{RT}$ to $850^{\circ} \mathrm{C}$;

basic pressure in UHV chamber is $10^{-8} \mathrm{mbar}$.

* Thermostatic enviromental cell for electrochemical hydrogen charging:

- $0.1 \mathrm{~N} \mathrm{NaOH}$ solution with $20 \mathrm{mg} / \mathrm{l}$ of $\mathrm{CS}\left(\mathrm{NH}_{2}\right)_{2}$

controlled potential of $-1.7 \mathrm{~V}$

pre-charged with hydrogen for $18 \mathrm{~h}$.

SEM and TEM techniques.

Figure 1: TDS apparatus.

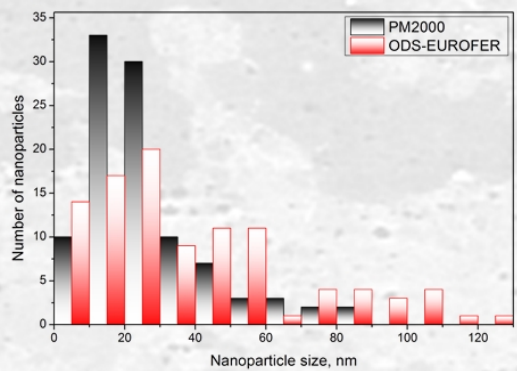

* Mean grain size of about $1 \mu \mathrm{m}$. PM2000. as shown in Figure 3.

Materials

\begin{tabular}{|l|c|c|c|c|c|c|c|c|c|c|c|}
\hline & \multicolumn{10}{|c|}{ Wt. \% } \\
\cline { 2 - 12 } & $\mathrm{C}$ & $\mathrm{Si}$ & $\mathrm{Mn}$ & $\mathrm{Cr}$ & $\mathrm{Ni}$ & $\mathrm{Mo}$ & $\mathrm{Al}$ & $\mathrm{W}$ & $\mathrm{V}$ & $\mathrm{Ti}$ & $\mathrm{Co}$ \\
\hline PM2000 & 0,017 & 0,01 & 0,09 & 19,3 & 0,03 & 0,08 & 4,8 & $<0,01$ & $<0,01$ & 0,5 & 0,15 \\
\hline Eurofer ODS & 0,088 & 0,02 & 0,4 & 9,2 & 0,03 & 0,02 & 0,006 & 1,14 & 0,18 & -- & 0,12 \\
\hline Eurofer 97 & 0,103 & 0,03 & 0,54 & 9,0 & 0,02 & 0,01 & 0,019 & 1,2 & 0,19 & -- & 0,17 \\
\hline
\end{tabular}

able 1: Chemical composition of EUROFER 97, ODS and PM200o steels.

Results, microstructure

Figure 2: Microstructure of (a) EUROFER 97, (b) ODS-EUROFER, and (c) PM200o obtained by TEM.
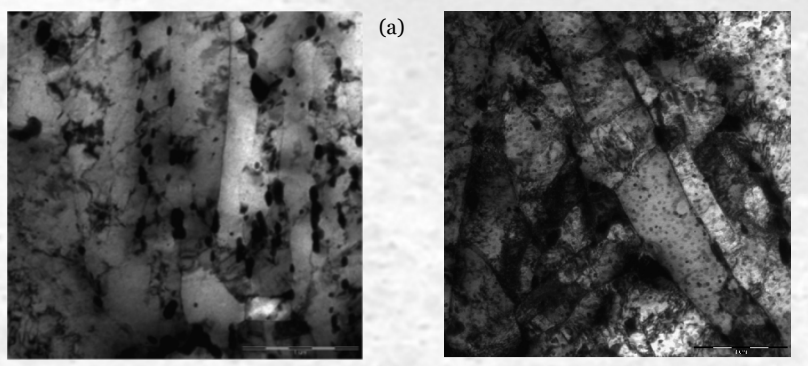

Figure 3: Nanoparticlc sice distibution for ODS EUROFER and PMeOr
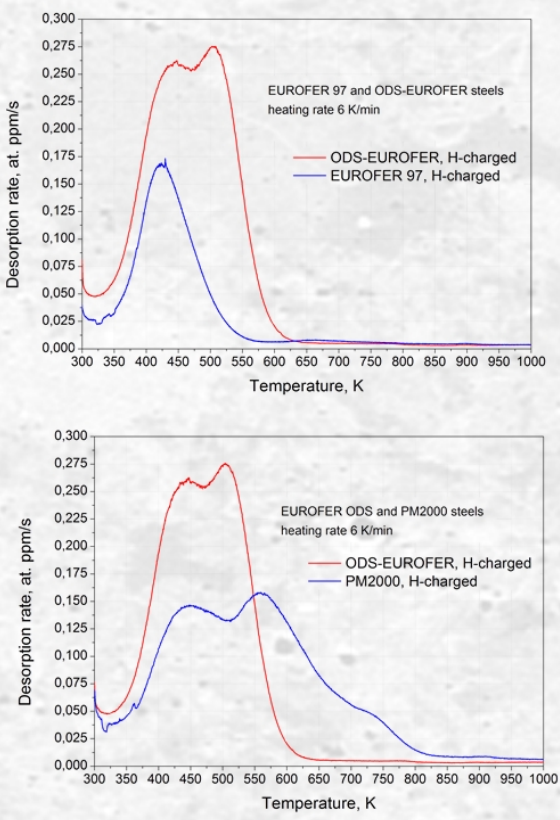

Figure 4: TDS spectra for hydrogen release from EUROFER 97, ODS-EUROFER and PM2000.
Elongated grain structure for all the studied specimens.

Mean density of ODS nanoparticles $\sim 1 \cdot 10^{22} \mathrm{~m}^{-3}$ for EUROFER ODS and $5 \cdot 10^{21} \mathrm{~m}^{-3}$ for

* The size of the nanoparticles in these materials varied from a few to hundreds nanometers.

*3. The size distribution analysis of the nanoparticles, having sizes smaller than $100 \mathrm{~nm}$, showed that PM200o has a smaller mean size of the nanoparticles than ODS-EUROFER, $10 \mathrm{~nm}$ vs. $20 \mathrm{~nm}$

. The EDS line scanning element profile analysis showed that in PM200o specimens nanoparticles with the sizes of hundred nanometers are aluminium oxide.

Results, TDS

The obtained thermal desorption spectra for three heating rates 2, 6 and 10 $\mathrm{K} / \mathrm{min}$ where used to characterize the binding states of hydrogen in the lattice and activation energies, $E_{\rho}$ of its release from the material.

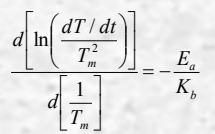

Where $\mathrm{dT} / \mathrm{dt}$ denotes the heating rate, $T_{m}$ is the temperature of the peak maximum, and $k_{b}$ is the Boltzmann constant.

\begin{tabular}{|l|c|c|c|}
\hline \multirow{2}{*}{} & \multicolumn{3}{|c|}{ Energy, eV } \\
\cline { 2 - 4 } & Peak 1 & Peak 2 & Peak 3 \\
\hline PM2000 & 0,20 & 0,20 & 0,38 \\
\hline Eurofer ODS & 0,26 & 0,35 & -- \\
\hline Eurofer 97 & 0,18 & -- & -- \\
\hline
\end{tabular}

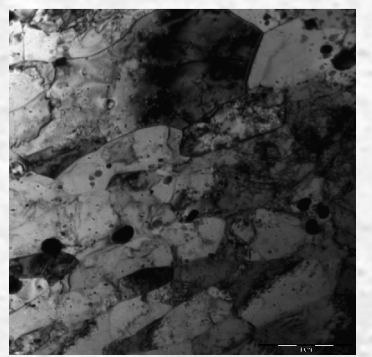

\section{Conclusions}

In this work the effect of strengthening of steels by yttrium oxide nanoparticles and their interaction with hydrogen is studied. Hydrogen uptake and effective activation energy of its diffusion and trapping are calculated from the thermal desorption spectra obtained for EUROFER 97, ODS-EUROFER, and PM2000 steels. It is demonstrated that embedding of nanoparticles leads to a significant increase of hydrogen uptake compared to the conventional steel, such as EUROFER 97. It is shown that hydrogen in ODS-EUROFER has the effective diffusion activation energy, which is 0.08 and $0.06 \mathrm{eV}$ larger than that in EUROFER 97 and PM200o, respectively. The high-temperature components of the TDS peaks of the studied ODS steels are suggested to reflect hydrogen de-trapping from the oxide nanoparticles. 